INPLASY

PROTOCOL

To cite: Yao et al. Effects of mirror therapy combined with electrical stimulation on balance and lower extremity motor recovery after stroke: a systematic review and metaanalysis of randomized controlled trials. Inplasy protocol 2020120049. doi: 10.37766/inplasy2020.12.0049

Received: 08 December 2020

Published: 09 December 2020

Corresponding author: Jun Yao

yaojun@njmu.edu.cn

Author Affiliation:

School of Health Policy and Management of Nanjing Medical University

Support: None.

Review Stage at time of this submission: Preliminary searches.

Conflicts of interest: None.

\section{Effects of mirror therapy combined with electrical stimulation on balance and lower extremity motor recovery after stroke: a systematic review and meta-analysis of randomized controlled trials}

Yao, J1; Wang, $\mathrm{H}^{2}$; Fang, $\mathrm{XY}^{3}$.

Review question / Objective: The aim of this systematic review and meta-analysis of randomized controlled trials is to evaluate the effects of mirror therapy combined with electrical stimulation on balance and lower extremity motor function recovery after stroke.

Condition being studied: Stroke is one of the primary causes of disability not only in middle-aged but also elderly people worldwide. Approximately $\mathbf{8 0} \%$ of survivors have an upper or lower extremity impairment after stroke. And two-thirds of people with lower extremity impairment are not able to walk independently soon, and after rehabilitation, only half have an independent walking function. Besides, the impaired walking ability can accentuate and maintain the burden of care for stroke patients. Therefore, the rehabilitation of impaired lower extremity motor function after stroke is a major issue.

INPLASY registration number: This protocol was registered with the International Platform of Registered Systematic Review and Meta-Analysis Protocols (INPLASY) on 09 December 2020 and was last updated on 09 December 2020 (registration number INPLASY2020120049).

\section{INTRODUCTION}

Review question / Objective: The aim of this systematic review and meta-analysis of randomized controlled trials is to evaluate the effects of mirror therapy combined with electrical stimulation on balance and lower extremity motor function recovery after stroke.

Condition being studied: Stroke is one of the primary causes of disability not only in 
middle-aged but also elderly people worldwide. Approximately $\mathbf{8 0} \%$ of survivors have an upper or lower extremity impairment after stroke. And two-thirds of people with lower extremity impairment are not able to walk independently soon, and after rehabilitation, only half have an independent walking function. Besides, the impaired walking ability can accentuate and maintain the burden of care for stroke patients. Therefore, the rehabilitation of impaired lower extremity motor function after stroke is a major issue.

\section{METHODS}

Participant or population: Participants aged $\geq 18$ years and having a clinical diagnosis of ischemic or hemorrhagic stroke with paresis of the lower limb will be included.

Intervention: Mirror therapy combined with one of the different modalities of electrical stimulation: neuromuscular electrical stimulation, functional electrical stimulation, afferent electrical stimulation, or electromyographic biofeedback.

Comparator: Conventional therapy, and mirror therapy or electrical stimulation isolated.

Study designs to be included: Randomized controlled trial

Eligibility criteria: Inclusion criteria: (i) randomized controlled trials in Chinese or English language; (ii) participants were aged $\geq 18$ years and had a clinical diagnosis of ischemic or hemorrhagic stroke with paresis of the lower extremity; (iii) intervention group: mirror therapy combined with one of the different modalities of electrical stimulation: neuromuscular electrical stimulation, functional electrical stimulation, afferent electrical stimulation, or electromyographic biofeedback; (iv) comparison group: conventional therapy, and mirror therapy or electrical stimulation isolated; (v) outcomes related to the lower extremity motor function and balance.
Information sources: We will search, with no time restrictions, the following databases for relevant English or Chinese literature: PubMed (MEDLINE), Web of Science, Embase, the Cochrane Central Register of Controlled Trials (CENTRAL), and CINAHL. The electronic database search will be supplemented by a manual search of the reference lists of included articles.

Main outcome(s): Fugl-Meyer AssessmentLower Extremity (FMA-LE) for lower extremity motor function.

Additional outcome(s): Berg Balance Scale (BBS) for balance.

Data management: Two authors will independently extract data. Any disagreement will be resolved by discussion until consensus is reached or by consulting a third author. The following data will be extracted: Study, Baseline characteristics, Sample size, Gender M/F, Ages, Duration of stroke, Paretic side Left/ Right, Stroke type ischemic/ hemorrhagic, Type of intervention, Treatment protocol, Outcome measures.

Quality assessment / Risk of bias analysis: Two authors screened each study to assess quality using the criteria of the Cochrane Handbook independently. The risk assessment criteria in the Cochrane Handbook are as follows: Random sequence generation, Allocation concealment, Blinding of personnel and participants, Blinding of outcome assessment, Incomplete outcome data, Selective reporting, Other bias.

Strategy of data synthesis: The Review Manager 5.4 software was also used for statistical analysis. Mean difference with a confidence interval of $95 \%(95 \% \mathrm{Cl})$ was used as the effect measure since the outcomes were continuous data, which were standardized to the same scale. A fixed-effect model was used when no heterogeneity was detected; a random model was used when heterogeneity was determined. The chi-square test and the $I^{2}$ statistic were used to determine the 
heterogeneity. An interpretation of the $\mathrm{I}^{2}$ is: 0\%-40\% might not be important; $30 \%-60 \%$ may represent moderate heterogeneity; $50 \%-90 \%$ may represent substantial heterogeneity; $75 \%-100 \%$ considerable heterogeneity. Forest plots were used to represent the results of the meta-analyses.

Subgroup analysis: The intervention of our study was the combined therapy of mirror therapy and electrical stimulation, and we presented it as an experimental group in all the analyses. We stratified the randomized trials according to the measurement instrument and then, the results were subdivided according to the type of comparison group. Three different subgroups were established as a comparison: conventional therapy, mirror therapy or electrical stimulation isolated. And they could provide a wider overview of the effects of the combined therapy.

Sensibility analysis: We conducted a sensitivity analysis to test the robustness of the results, removing studies that we assessed to be of lower methodological quality.

Country(ies) involved: China.

Keywords: Electric stimulation, mirror therapy, rehabilitation, stroke.

Contributions of each author:

Author 1 - Jun Yao.

Email: yaojun@njmu.edu.cn

Author 2 - Hao Wang.

Email: blake.h.wang@outlook.com

Author 3 - Xiaoya Fang.

Email: fangxiaoya99@163.com 